\author{
Mirosław Lenart* \\ Instytut Nauk o Literaturze, Uniwersytet Opolski \\ Archiwum Państwowe w Opolu
}

\title{
Osservazioni sulla cultura delle ville venete in Polonia nel XVI secolo ${ }^{1}$
}

\section{Some Remarks Concerning Venetian Mansions in the 16th-century Poland}

\begin{abstract}
This paper presents the inter-relationships between the Poles arriving at Padova for University studies or actively present in the Venetian Republic during the Renaissance and the representatives of the Serenissima intellectual elite. The author is particularly interested in the aspects of the contacts that touch upon the humanist culture developed in the suburban mansions, which constituted the characteristic feature of the culture at the turn of the $15^{\text {th }}$ century. The paper examines the arguments in support of the thesis that the numerous presence of the incomers from the Polish-Lithuanian Commonwealth had a decisive influence on the cultural choices made by Polish and Lithuanian gentry in regards to construction of the suburban residences and their ambitions to
\end{abstract}

* lenart@uni.opole.pl, Lenart, M. (2019). Osservazioni sulla cultura delle ville venete in Polonia nel XVI secolo. Annales Collegii Nobilium Opolienses, 8, pp. 1141.

1 Il presente articolo è stato pubblicato precedentemente negli atti del convegno: Corti rinascimentali extraurbane. Un modello di cultura tra Italia e Polonia. Atti del Convegno Internazionale (Altivole - Castelfranco Veneto - Maser - Vedelago - Mira - Padova - Luvigliano 16-18, settembre 2013). Podmiejskie dwory renesansowe. Wzorzec kulturowy we Włoszech i w Polsce. Materiały międzynarodowej konferencji naukowej (Altivole - Castelfranco Veneto - Maser - Vedelago - Mira - Padova - Luvigliano 16-18 września 2013), a cura di M. Lenart, M. Wrana, Opole 2016 r. (Archiwalne źródła tożsamości, 1; Natio Ultramontana. Commentarii et Studia, 1). 
introduce into those spaces some elements of art and literary culture adopted from the environment in which they had lived in Italy.

Keywords: suburban Renaissance courts, Venetian mansions, suburban residences in 16th-century Poland

Gli studi sulla cultura delle residenze rinascimentali extraurbane fino a oggi realizzati, in rare occasioni hanno messo a confronto il sapere teorico dei testi, soprattutto letterari, con gli spazi concreti. Per questo molto importante e facente ben sperare per la scoperta futura di nuovi campi scientifici si considera l'apertura verso le informazioni che arrivano non solo dalla letteratura, ma anche dalle analisi dei monumenti artistici e architettonici che si sono conservati, soprattutto di quelli che fino a oggi sono ancora situati in un circondario caratteristico ${ }^{2}$. In questo caso il territorio dell'antica Repubblica di Venezia sembra essere un ambito assai generoso per ciò che riguarda l'introduzione di interessanti osservazioni e analisi, in quanto, proprio all'interno dei suoi confini si sono conservate delle opere architettoniche, le quali spesso si presentano ancora nel loro stato originale o perlomeno si trovano in un tale stato che ci permette di stimolare l'immaginazione delle persone che desiderano avvicinarsi all'importanza del significato della realizzazione delle residenze extraurbane per la cultura del rinascimento. Inoltre si tratta di presupposti e di costruzioni molto antiche legate in modo specifico all'attività di famosi rappresentanti dell'umanesimo, il che in questa sede ci interessa in modo particolare. È inoltre importante sottolineare l'importanza del primo periodo di affermazione dell'idea delle ville situate lontano dalla città, in un ambiente rurale che solo in un successivo momento è stato trasformato in una forma precisa, soprattutto grazie al genio di Andrea Palladio. La sua influenza sullarchitettura fu così forte e allo stesso tempo duratura al punto che la villa viene oggi intesa soprattutto nella sua dimensione estetica, sebbene per secoli avesse svolto un ruolo chiave nellorganizzazione politica economica e culturale dello

2 In questo campo gli studi sono decisamente più sviluppati. Vedasi il catalogo della mostra: Andrea Palladio e la villa veneta da Petrarca a Carlo Scarpa, Vicenza, Museo Palladio in palazzo Barbaran da Porto, 5 marzo-3 luglio 2005, a cura di G. Beltramini, H. Burns, fotografie delle ville di Pino Guidolotti, Venezia 2005. 
Stato veneziano. Proprio l'aspetto della vita culturale, indissolubilmente legato alla villa veneziana, attirò tantissime personalità d'oltralpe, considerazione che sembra degna di una più profonda riflessione, in quanto l'influenza sulla forma dei gusti e delle scelte, era forte particolarmente laddove il ruolo dei proprietari terrieri era particolarmente significativo. Naturalmente ciò che nel nostro caso più ci interessa è l'acquisizione di elementi di questo settore della vita degli umanisti italiani da parte dei polacchi, in quanto essi costituivano un gruppo interessato in modo naturale a tutto ciò che sembrava loro essere vicino. Le ispirazioni che arrivarono dal contatto con questo fenomeno tipico di questo territorio erano in questo caso caratterizzate da un aspetto più spirituale che materiale e si richiamavano più alla ricerca delle soluzioni personali nella scoperta della propria identità nazionale con una continua ricezione dellapprendimento della cultura del rinascimento. Riguardo alla lunga durata della cultura delle ville extraurbane e il loro influsso sull'architettura, arte e cultura, con le quali oggi sono confrontate, di notevole importanza era per la realtà polacca soprattutto la fase iniziale della loro trasformazione, che avvenne nella prima metà del XVI secolo. Si tratta del periodo meno conosciuto sia in Polonia che in Italia, in quanto il fenomeno della creazione di sedi fuori città che possedevano un loro carattere specifico si interpreta oggi in un contesto temporale ben più ampio, grazie al numero delle costruzioni conservatisi. Teniamo a ricordare che solo nell'attuale territorio del Veneto e del Friuli si sono conservate circa quattromila residenze di tal tipo, le quali vengono recepite come elemento del paesaggio e allo stesso tempo come punto di riferimento storico nell'ascrivere un'identità a questi territori.

Notevolmente differente è la situazione che si presenta sul territorio polacco, dove le realizzazioni architettoniche legate in qualche modo all'idea della realizzazione di sedi extraurbane sulla base dei modelli rinascimentali si sono conservate in uno stato minimo, come tra l'altro altre testimonianze della cultura materiale di allora presenti in queste zone. Nel campo della ricerca tematica di relazioni reciproche tra la Polonia e l'Italia questi fatti hanno un'importanza fondamentale. La mancanza di un corposo materiale di comparazione, al fine di ricostruire i rapporti di scambio tra i due paesi, porta gli studiosi a ricercare le fonti nelle documentazioni presenti soprattutto nelle biblioteche e negli archivi.

Lambientazione dei fenomeni che ci interessano in un dato periodo e in concrete aree, anche se dobbiamo aiutarci con una buona dose di 
immaginazione in questo campo, permette una migliore comprensione dell'avventura intellettuale con cui ebbero a che fare gli allora viaggiatori e studenti provenienti dalla Polonia. Il territorio della Repubblica di Venezia deve essere trattato in modo assai specifico, in quanto proprio in esso avvenne il contatto di massa dei polacchi con l'intenso sviluppo della villa, che a suo tempo era il risultato dell'espansione dei veneziani sui quei territori della terraferma ${ }^{3}$, che fino ad allora non erano stati sfruttati. Ciò avvenne verso la fine del XV e l'inizio del XVI secolo.

Il posto in cui si trovavano i rappresentanti della nazione polacca era comunque Padova, insieme alla sua università che con la sua fama attirava studenti da tutta Europa, una città che era tra l'altro teatro di altre trasformazioni non meno importanti. L' ateneo patavino giocava un ruolo importante nella politica della Serenissima, la quale conduceva una propaganda in larga scala al fine di presentare un paese moderno e libero, dove fiorivano arte e cultura. Non molto tempo dopo la riapertura dell'Università, avvenuta nell'anno 1517 (l'ateneo era stato chiuso per 8 anni in virtù dello svolgimento del conflitto con la lega di Cambrai), gli studenti polacchi ebbero occasione di comprendere come questo centro del sapere, che Erasmo da Rotterdam riteneva il migliore d'Italia ${ }^{4}$, si stesse dinamicamente sviluppando.

Dal momento della ripresa delle attività, si decise per una subitanea riacquisizione dell'antico ruolo dell'ateneo e quindi, usando un'espressione dellepoca, «ritornar il Studio in quel florido stato che l'era solito esser $»^{5}$. L' apertura dell'università era legata al cambio di potere all'interno dell'ateneo ${ }^{6}$. Tale cambiamento consisteva in un graduale passaggio di potere nelle mani del magistrato di Venezia attraverso un totale controllo sull'università da parte dell'organo dei Riformatori dello studio di Pado$v a$, che con il passare del tempo riuscì a subordinare tutte le istituzioni che avevano importanza a livello culturale, non solo a Padova, ma anche

3 Vedi M. Lenart, Patavium, Pava, Padwa. Tło kulturowe pobytu Jana Kochanowskiego na terytorium Republiki Weneckiej, Warszawa 2013, pp. 13-37 («Studia Staropolskie. Series Nova», tom XXXIII (LXXXIX)).

4 Vedi P. Del Negro, L’età moderna, in: L’Università di Padova. Otto secoli di storia, a cura di P. Del Negro, Padova 2002, pp. 40-41.

5 Ivi, p. 40.

6 Vedi l'università di Padova che all'inizio dipendeva dal vescovo di Padova, durante gli anni di gorverno della Serenissima era amministrata dai suoi rappresentanti in città. Cfr. A. Da Mosto, L'Archivio di Stato di Venezia, Roma 1937, p. 217. 
a Venezia: dalla stamperia alla scuola di diversi livelli ${ }^{7}$. Si trattava di una struttura provvisoria e temporale già istituita nel 1516, la quale comunque dal 22 settembre 1528 era diventata l'organo che avrebbe amministrato l'università fino alla caduta della Repubblica di Venezia, quindi per i seguenti tre secoli. Questo periodo fatto di dinamici cambiamenti che riguardavano sia l'università sia lo sviluppo della Repubblica di Venezia, si riconduce alla presenza di rappresentanti della nazione polacca ritenuti di una certa importanza. Si tratta di personalità come Klemens Janicki, Samuel Maciejowski, Wojciech Kryski, Jan Derśniak Andrzej Kostka, Petrycy Nidecki, Jan Kochanowski, Jan Zamoyski e molti altri. Le loro esperienze comuni acquisite sul territorio della Serenissima costituivano un forte legame, ma anche un canale di comunicazione specifico che permetteva un contatto tra i gruppi anche dopo il ritorno in Polonia ${ }^{8}$. Questo dialogo riguardava allora un nutrito numero di persone molto influenti presso la corte e le strutture della chiesa. Stanisław Windakiewicz, nel libro pubblicato nel 1922, quando il Gymnasium Patavinum festeggiò un importante anniversario della sua esistenza scrisse che

in base agli «Acta Universitatis legistarum» a Padova e alle identificazioni del catalogo dei nomi che vi figurano con le fonti polacche, si può dimostrare che 49 futuri vescovi ed abati, 39 palatini e castellani, 56 magistrati provinciali e deputati alla dieta, erano stati allievi della facoltà di legge a Padova. Alcuni studenti padovani occuparono poi cariche importanti nel senato e nel parlamento polacco. Anche fra i giudici, deputati ai tribunali e agli ufficiali fiscali, abbiamo trovato 30 ex-allievi della facoltà di giurisprudenza di Padova9.

7 Per l' esattezza Riformatori sopra el Studio de Padoa così suonava il nome dell'istituzione in lingua veneta. Essa continuava i lavori della precedente commissione composta da tre senatori che venivano chiamati deputati a redur lo studio. Vedi P. Del Negro, L' età moderna..., cit., p. 40.

8 M. Lenart, Studenti polacchi a Padova e loro mecenatismo in patria, in: Mecenati, artisti e pubblico nel Rinascimento. Atti del XXI Convegno Internazionale Chianciano Terme-Pienza, a cura di L. Secchi Tarugi, Firenze 2011, pp. 501-511 (Quaderni della Rassegna, 68).

9 S. Windakiewicz, I polacchi a Padova, in: Omaggio dell'Accademia Polacca di Scienze e Lettere all'Università di Padova nel settimo centenario della sua fondazione, Cracovia 1922, p. 14; vedi anche idem, Padwa, Kraków 1891, pp. 23-24: «na bazie „Acta Universitatis legistarum” przechowywanych w Padwie oraz identyfikacji 
Dai dati generali risulta che solo nel XVI secolo a Padova abitavano 1600 polacchi senza tener conto di quelli che erano arrivati a Padova solo per conoscere la vita culturale di quella città, come era di moda allora in tutta la Polonia. Almeno una parte di questa schiera era composta da personalità più o meno importanti, per le quali l'urbanità significava soprattutto un contatto con la cultura italiana; avevano infatti l' occasione di passeggiare per i giardini delle residenze veneziane, il che era risultato di contatti stabili e duraturi tra le due parti con i rappresentanti dell' elite intellettuale italiana, resiedente nel territorio della Repubblica di Venezia.

Proprio essi, dopo il ritorno in Polonia, svilupparono la loro carriera nelle strutture della chiesa, ma anche presso la corte reale, rimanendo comunque sotto la preponderante influenza della cultura che fioriva a Padova, dalla quale attingevano un proprio codice di comprensione tra loro stessi ${ }^{10}$. Una parte di loro (Kryski, Derśniak i Kostka) ebbero il merito di aver creato nella Padova studentesca "l'Accademia tra i polacchi" che venne realizzata negli ultimi anni del quarto decennio del XVI secolo ${ }^{11}$. La sede dell'accademia era situata nel così chiamato contubernium Polonorum, che si trovava in una casa presa in affitto non lontano dalla basilica di Sant'Antonio vicino alla 'Crosera del Santo' ${ }^{12}$. Proprio tale posto che riuniva l' elite degli studenti polacchi e allo stesso tempo fungeva da punto di apertura dei contatti con i rappresentanti di altre nazioni, diventò il simbolo dei legami reciproci basati sulle comuni acquisizioni culturali ricevute nella città di Antenore. Il ricordo di una casa spaziosa e dipinta ${ }^{13}$ in cui abitavano i polacchi permette inoltre di

osób z katalogu imion tam figurujących ze źródłami polskimi można wykazać, że 49 przyszłych biskupów i opatów, 39 wojewodów i kasztelanów, 56 sędziów grodzkich i posłów na sejm było studentami prawa w Padwie. Niektórzy studenci padewscy zajmowali później ważne funkcje w senacie i parlamencie polskim. Także wśród prawników, delegatów do sądów oraz urzędów skarbu, znaleźliśmy 30 byłych studentów prawa w Padwie».

10 M. Lenart, Patavium, Pava, Padwa..., cit., pp. 38-72.

11 Ivi, pp. 60-63.

12 Ivi, pp. 44-52.

13 In un documneto da archivio del 5 luglio 1543 si viene a conoscenza che Francesco Corona dipinse la casa di Giacomo Cicuta, dove abitavano i polacchi: «Il pittore Francesco Corona, q. Girolamo, abitante a s. Matteo, afferma d'aver dipinto durante il tempo in cui Giacomo Cicuta, dalmata, era rettore dei Giuristi a Padova, delle mazze ed anche la facciata della sua casa "quando io depensi poi la fazà della casa sui no ghel visti più”» (Archivio di Stato di Padova, ASP, Notarile, t. 4068, c. 122). 
imbastire un tessuto di contatti che ci aiuteranno a fare i primi passi nella ricostruzione di uno degli aspetti della loro avventura ideale con il mondo delle ville venete.

Testimone del contratto di affitto stipulato nel 1545 fu Lazzaro Bonamico di Bassano (morto nel1552), famoso grecista e insegnante assai amato dalgi studenti polacchi e, forse proprio per questo, mancato precettore di Sigismondo Augusto, erede al trono polacco. Egli, in una lettera mandata a Pietro Bembo, raccomandava Piotr Kmita, uno dei più ricchi e più influenti cittadini della Rzeczpospolita. Riguardo alle sue relazioni con i polacchi disse: "Cum Polonorum gente magna mihi intercedit gratia, multis inter nos officiis et conciliata et aucta» (con i polacchi mi unisce una grande amicizia, sempre più rinforzata da molti atti di aiuto reciproco) ${ }^{14}$. Questa testimonianza dimostra quali profonde relazioni avesse Bonamico ${ }^{15}$ con i polacchi, relazioni basate su un grado di reciprochi favori dei quali ci possiamo solo immaginare. Cerchiamo di porre attenzione al fatto che egli stesso poteva essere per i polacchi non solo un modello di umanista, ma anche in qualità di uomo d'affari, essendo infatti legato ai possedimenti della provincia padovana ${ }^{16}$. La lettera in questione suscita il nostro interesse soprattutto per la citata figura di Piotr Kmita (morto nel 1553). Questo discepolo della corte di Massimiliano I, dove aveva passato la sua gioventù, lasciò traccia di sé nella storia sia come mecenate della cultura e la sua corte presso il castello di Wiśnicz era annoverata tra i più importanti centri del rinascimento polacco, in quanto vi si riunivano eminenti intellettuali polacchi tra i quali vi erano avvocati, scrittori e poeti ${ }^{17}$. Purtroppo del castello di Mokrsko Górne, probabilmente fatto riedificare dallo stesso Kmita seguendo i dettami delle residenze fortificate italiane, ci restano solo le rovine. Grazie a queste osservazioni, non ancora confermate, a causa della mancanza di fonti che potrebbero fornire maggiori particolari riguardo all'attività del magnate polacco, per noi degne di inte-

14 Vedi F. Piovan, Per la biografia di Lazzaro Bonamico ricerche sul periodo dell'insegnamento padovano (1530-1552), Trieste 1988, pp. 89.

15 Vedi M. Lenart, Lazzaro Bonamico - „buon' amico con tutti” - i jego relacje z Polakami, in: Przyjaźń w kulturze staropolskiej, a cura di A. Czechowicz, M. Trębska, Lublin 2013, pp. 117-126 («Studia i Materiały do Dziejów Literatury Wczesnonowożytnej», 2).

16 Ivi, pp. 119-121.

17 H. Barycz, Kulturalna działalność Piotra Kmity, «Rocznik Towarzystwa Przyjaciół Nauk w Przemyślu na rok 1925», t. 6, pp. 3-72. 
resse, possiamo meglio comprendere il significato dei testi scritti a mano di un poeta, del quale per un certo periodo si prese cura come mecenate: di Klemens Janicki (Clemens Ianicius 1516-1543) ${ }^{18}$.

Se invece ci rivolgiamo a concrete opere poetiche bisogna rammentare ancora una volta Bonamico, ricordato in occasione della presentazione della biografia di questo polacco appassionato di cultura antica. È proprio grazie al suo aiuto del tutore degli studenti polacchi, Janicki, afflitto da una malattia e privo di grandi mezzi finanziari (ricordiamo che egli proveniva da una famiglia contadina) potè iniziare il suo percorso di studi all'università. Janicki arrivò a Padova nel 1538 e vi rimase per più di due anni. Sebbene non fosse trattato di un lungo soggiorno, egli riuscì comunque a ricevere dal podestà Marcantonio Contarini il diploma di dottore in artibus et philosofia. Era comunque dottore bullatus, non academicus né promotus. Ma la cosa più importante per lui e per la storia della cultura polacca fu l'aver ricevuto il lauro poetico e il titolo di poeta laureatus. Riconoscente per l'aiuto ricevuto, dedicò a Bonamico due elegie, pubblicate più tardi nella raccolta Tristia VI e Variae elegiae VIII, dando conferma della sua amicizia con il professore delle lingue classiche, al quale in buona parte era riconoscente per la sua capacità di comporre poesie, così come per le cure mediche e persino per l'appoggio finanziario. Ė molto probabile che a Padova Janicki entrò in contatto con Pietro Bembo. In ogni caso il poeta polacco fa riferimento a questa indiscutibile autorità dell' epoca in una delle sue elegie e in un epigramma (Variae elegiae IX, Epigrammata LII). Per quanto il primo testo sia stato scritto subito dopo la nomina cardinalizia di Bembo nel 1539, la sua tematica si concentra sull' esaltazione della vita a Padova e nei suoi dintorni ma anche sulle virtù dell'umanista, il che fa del componimento un' opera a carattere occasionale ${ }^{19}$; molto più interessante è invece il componimento dalla raccolta dell' Epigramma II. Tale testo, dedicato in questo caso al mecenate Piotr Kmita e composto in Polonia dopo il suo ritorno a Cracovia, contrappone la delicata foglia di alloro che può essere distrutta dai venti, dal freddo, dalla neve e dalla pioggia, a tutto il

18 E. Kotarski, Klemens Janicki, in: Pisarze staropolscy. Sylwetki, a cura di S. Grzeszczuk, Warszawa 1991, pp. 377-406.

19 J. Ślaski, Wokół literatury włoskiej, węgierskiej i polskiej w epoce renesansu. Szkice komparatystyczne, Warszawa 1991, p. 159; vedi anche R. Krzywy, Podróże do nowej Arkadii. Wizja Włoch w elegiach Klemensa Janicjusza, «Terminus», XIII (2011), fasc. 24, p. 94. 
bosco fatto da alloro riparato da una siepe che si trovava nel giardino di Bembo. È possibile che si tratti del giardino del chiostro di Villa Bozzi (Nonianum) a Santa Maria di Non, nelle vicinanze di Padova, celebrato nelle strofe poetiche anche dal proprietario stesso (Sonetto LIV) ${ }^{20}$. L' epigramma qui citato ha come fine convincere il mecenate polacco, in una forma assai raffinata, a fornirgli un ulteriore aiuto. Alcuni pensano che Janicki fosse ospite del ricovero che si trovava nelle vicinanze della città, e che dei piaceri del giardino che l'umanista possedeva a Padova presso la sua residenza fosse venuto a conoscenza soltanto per sentito dire. Per noi il fatto più importante resta che l'immaginazione di tale luogo o altri ad esso simili, doveva essere un segno tangibile per Kmita che aveva viaggiato per le terre italiane e corrispondeva con Bembo ${ }^{21}$. Possiamo comunque aggiungere che i giardini degli umanisti padovani svolgevano differenti funzioni che attiravano l'attenzione degli studenti polacchi. Siamo a conoscenza del fatto che il benemerito medico Marcin di Urzędów nel 1538 ricevette a Padova il dottorato in medicina e che aveva raccolto erbe medicinali nel giardino di Bembo (sospettiamo che si trattasse del giardino che si trovava in città) e lo stesso fece un polacco meno conosciuto che portava il nome di Erazm ${ }^{22}$. I polacchi pensano di vedere in questi giardini del chiostro dei riferimenti all'antico non solo attraverso la trasfigurazione della realtà descritta nella letteratura. Il giardino come locus amoenus era per loro nello stesso tempo un posto dove potevano entrare in contatto con il mondo antico, presente nel suo spazio grazie alle raccolte di resti romani che a Padova non venivano collezionate solo da Bembo, ma anche da altri esperti dell'antichità vicini ai polacchi come Marco Mantova Benavides oppure Alessandro Magi da Bassano e anche Niccolò Leonico Tomeo.

Di come il ricordo delle residenze extraurbane sia rimasto nella memoria degli studenti polacchi che avevano abitato a Padova può essere ulteriore conferma un altro frammento del secondo libro degli epigrammi, dove si trova un verso di Janicki, indirizzato a Just Ludwik Decjusz, nel quale celebrava la sua villa, edificata nel 1535 nelle vicinanze di Cracovia:

20 Vedi Rime di m. Pietro Bembo, Milano 1808, p. 49. Il sonetto LV, al quale ci richiamiamo, inizia dalle parole "Lieta e chiusa contrada, ov'io m'involo».

21 M. Lenart, Lazzaro Bonamico..., cit., pp. 121-122.

22 L. Ćwikliński, Klemens Janicki poeta uwieńczony (1516-1543), Kraków 1893, p. 64 . 
Sic me structa recens delectant atria, sic me Hortus et a pulchris vitibus umbra iuvat; Sic quae per colles velut errat silva propinquos, Sic capiunt vitrei me recreantque lacus. Libera libertas libuit quod cuique bibendi, Sic placet Ausonii dulcis alumna soli ${ }^{23}$.

La residenza extraurbana e il suo circondario non devono, secondo Janicki, avere nulla da invidiare all'Ausonia, ciò attira la nostra attenzione soprattutto nel carattere del solo paragone. Dalla poesia del poeta morto prematuramente ma insignito del lauro poetico a Padova, sappiamo anche che egli strinse rapporti damicizia con Daniele Barbaro (1514-1570) al quale dedicò un altro epigramma (Epigrammata XLIX, L). Vi inserì un tipo di polemica sul tema dellamore in riferimento alla poesia di Barbaro, il che può significare una certa confidenza tra i due poeti. Ci preme ricordare che il futuro cardinale, coetaneo del polacco, ma anche proprietario di una delle prime ville palladiane, strinse $\mathrm{a}$ Padova contatti con le personalità più famose e influenti, con cui fondò l'Accademia degli Infiammati ${ }^{24}$, la quale era attiva nei primi quarantanni del XVI secolo ${ }^{25}$, e i cui membri scelsero come sede la residenza del mecenate padovano Alvise Cornaro ${ }^{26}$.

Proprio da essa i polacchi presero spunto quando decisero di creare la succitata Accademia tra i polacchi, che va annoverata come un'iniziativa eccezionale tra gli studenti ${ }^{27}$. Della sua esistenza veniamo a sapere

23 C. Ianicii, Carmina, edidit, prefatione instruxit, annotationibus illustravit L. Ćwikliński, Cracoviae 1930, p. 260 («Corpus Antiquissimorum Poetarum Poloniae Latinorum usque ad Ioannem Cochanovium», 6); L. Ćwikliński, O wawrzynie doktorskim i poetyckim Klemensa Janickiego, Kraków, 1919, «Rozprawy», t. 58; n. 6.

24 Vedi G. Vedova, Biografia degli scrittori padovani, Padova 1832, I, pp. 491-492; M. Maylender, Storia delle accademie d'Italia, III, Bologna 1929, p. 266; V. Vianello, Il letterato, l'accademia, il libro contributi sulla cultura veneta del Cinquecento, Padova 1988, pp. 47-70; A. Andreoni, Benedetto Varchi all'Accademia degli Infiammati. Frammenti inediti e appunti sui manoscritti, «Studi Rinascimentali», 3 (2005), pp. 29-44.

25 M. Lenart, Patavium, Pava, Padwa..., cit., pp. 40-46; 113-119.

26 Ivi, pp.113.

27 Cfr. M. Lenart, Epitaphium Cretcovii świadectwem kontaktów padewskich Jana Kochanowskiego z otoczeniem Alvisa Cornara?, in: Twórczość Jana Kocha- 
da un'unica fonte, quale il Dworzanin Polski, un rifacimento de Il libro del Cortegiano di Castiglione, scritto da Łukasz Górnicki, e pubblicato a Cracovia nel 1566. Górnicki, raffinato umanista, le cui capacità letterarie e di traduzione erano un fatto eccezionale non solo in Polonia, $\mathrm{ma}$ anche in tutta l'area slava ${ }^{28}$, riuscì con grande abilità ad avvicinare al suo mondo l'opera legata alla lontana Urbino e alla corte di Montefeltro. A questo punto bisogna sottolineare che la versione polacca è nella sua completezza ambientata nel palazzo dei vescovi di Cracovia, il che conferma il carattere del lavoro di Górnicki, il quale attinge al modello italiano, ma lo adatta alla realtà conosciuta dai lettori polacchi. Forse bisognerebbe anche sottolineaere in questo caso il trattamento delle tematiche affrontate in un modo spesso diverso dall' originale, dove il paragone di entrambi i mondi perde di significato, mettendo al primo posto la continuazione della cultura dell'umanismo dominante in Europa. Ritornando alla residenza è lecito ricordare che essa venne voluta dal vescovo e dal gran cancelliere della corona Samuel Maciejwski (morto nel 1550) nelle vicinanze di Cracovia, allora capitale polacca, soprattutto con il fine di assolvere a necessità diplomatiche. Il posto scelto era chiamato Prądnik, ricco di giardini realizzati secondo il modello italiano, costituiva lo sfondo ideale per coloro che erano desiderosi di dibattere. Nelle parole di introduzione del vescovo indirizzate a Wojciech Kryski, buon conoscitore dell'Italia, ispiratore e fondatore dell'Accademia, leggiamo tra le altre cose:

Panie Kryski, ku WM. ja mówię, któryś podobno lepiej niż kto iny włoskiej ziemi świadom, pokuś WM. tego, a tak jakoś był powodem do Akademiej Padewskiej miedzy Polaki (jako ja mam sprawę), tak i tu niechajby sie takowe gry od WM. poczęly. Masz oto WM. równe towarzystwo, pana Wapowskiego, pana Myszkowskiego, pana Derśniaka, którzy tego WM. radzi

nowskiego w kontekście nowołacińskiej literatury europejskiej i polskiej, a cura di G. Urban-Godziek, Kraków 2010, pp. 64-75; idem, Patavium, Pava, Padwa..., cit., pp. 60-63.

28 T. Ulewicz, L'enigmatica Accademia degli studenti polacchi a Padova (negli anni 1547-1549 ca.), "Atti e Memorie dell'Accademia Patavina di Scienze, Lettere ed Arti», 100 (1987-1988), pp. 87-93. 
pomogą. I Kostka mój, chocia młody, jednak w tej mierze może sie popisać niegrubie ${ }^{29}$.

E subito dopo, torna su questo tema dicendo:

bo kiedy się pan Wapowski, pan Kostka, pan Derśniak rozumy swemi do tego przyłożą, wskrzesi sie znowu ona ich Academia Padewska, ktorąśs WM. teraz wspominać raczył, a ze mnie gotowego jednego ucznia mają $z^{30}$.

Il frammento qui presentato conferma non solo l'innegabile significato che il soggiorno italiano, soprattutto padovano, ha avuto per gli intellettuali polacchi chiamati "padewczycy" [padovani, n.d.t.] in quanto essi si distinsero non solo presso la corte reale, ma anche in generale nella cultura polacca di quel tempo. Non meno importante è il fatto che il richiamo ai significati comprensibili nell'ambito dell'umanesimo italiano sia legato con la villa extraurbana, nella quale troviamo tutti i riferimenti che ci interessano, villa simile a quella che venne edificata verso la fine XV secolo fuori Padova, a Luvigliano, grazie all'iniziativa del vescovo Jacopo Zeno.

Prima ditornare a questo posto che ha ispirato significativi cambiamenti all'inizio del secolo successivo, restiamo ancora presso i giardini della Serenissima. Le attività intraprese con grande slancio dal Cinquecento, aventi come scopo il miglioramento dei terreni, e nel 1501 legate alla realizzazione della Magistratura delle Acque portarono all'acquisizione di gran-

29 Ł. Górnicki, Dworzanin polski, in: idem, Pisma, a cura di R. Pollak, Wrocław 1954, pp. 65-66.

30 Ivi, pp. 67-68. Trad. it: «Signor Kryski, è a voi, Vossignoria, che parlo io, a voi che siete, meglio degli altri, un vero conoscitore della terra italiana, abbiate coraggio di farlo e così come siete stati ispiratore dell'Accademia tra i Polacchi... (come io ne ho ragguaglio), che anche qui si provi a intraprendere, da Vossignoria, tale attività! Eccovi a disposizione vostra, Vossignoria, la compagnia migliore tra cui il signor Wapowski, il signor Derśniak, i quali vi ci aiutano volentieri, Vossignoria. Anche il mio Kostka, nonostante che sia giovane vi può essere d'aiuto formidabile. [...] E quand’è che il signor Wapowski e il signor Kostka ci aiuteranno con i loro intelletti, si riapre di nuovo quella loro Accademia Padovana, che Vossignoria ha degnato ora di ricordare ed io sono gia pronto ad essere un nuovo studente di questa accademia». 
di terreni che in precedenza non solo non erano abitabili, ma nemmeno coltivabili. Tutte queste azioni presero uno slancio ancora maggiore nel 1556, quando iniziò a funzionare il Magistrato ai Beni Inculti, istituzione creata per il controllo e l'appianamento degli interessi pubblici e privati, legati all'acquisto e alla coltivazione della terra. L' acquisto di terreni diventa per i veneziani non solo un simbolo di potere, ma anche permette maggiori entrate e soddisfa i sempre crescenti bisogni culturali dellaristocrazia e della borghesia ${ }^{31}$. Questi così grandi cambiamenti nella politica economica dello Stato veneziano non incontrarono, perlomeno all'inizio, il favore di buona parte del patriziato che rimaneva su posizioni tradizionali. Il già citato, nel contesto dell'Accademia degli infiammati, Alvise Cornaro, faceva parte di quelli che in modo coerente rispondevano alla concezione della sancta agricoltura, come la chiamò in una letterea a Sperone Speroni $^{32}$, unattività nobile e morale superiore al commercio, già lodata da Virgilio nelle Georgiche e da altri scrittori dell'antichità. La cultura di quei tempi deve proprio a Alvise Cornaro la concezione dellamministrazione che permette di utilizzare i proventi destinati alla realizzazione della villa, la quale non solo risponde a una esigenza di amministrazione dei beni, ma diventa anche un posto degno di un ricco proprietario con grandi aspirazioni culturali. Il fatto che vennero apprezzate le sue capacità nel campo dell'amministrazione trova conferma nel fatto che il cardinale Francesco Pisani, vescovo di Padova negli anni 1524-1555, gli concesse in amministrazione i beni episcopali che secondo le stime contavano 4000 campi, cioé poco meno di 2000 ettari.

Proprio Cornaro contribuì in modo significativo alla trasformazione della sede dei vertici della chiesa padovana nella succitata Luvigliano, dove venne edificata una residenza chiamata oggi Villa dei Vescovi. Alla realizzazione di tale opera prese parte una squadra di artisti con i quali Cornaro aveva costruito e abbellito i suoi beni, soprattutto quelli che si trovavano nel terreno di Padova. I lavori di ristrutturazione del gigantesco complesso del palazzo-giardino di Luvigliano, terminati nel 1524

31 Il più importante trattato sul praedium rusticum è: A. Lollio, Lettera in laude della villa, Ferrara, F. Rossi, 1540 (Venetia 1544), e il dialogo La villa del lombardo Bartolomeo Taegio, pubblicato a Milano (F. Moscheni, 1599). Segnaliamo inoltre i manuali: La nuova, voga, et dilettevole villa di G. Falconi (Milano 1559); Le dieci giornate della vera agricoltura di A. Gallo (Venezia 1566).

32 M. Muraro, Civiltà delle ville Venete. Conferenza tenuta il 23.10.1964 alla Hertziana di Roma, Venezia 1964, pp. 20, 25. 
e che comprendevano anche le decorazioni della composizione del paesaggio in cui il palazzo era inserito, fecero una grande impressione alle personalità di allora. Si tratta di un esempio di una residenza di campagna, nella quale l'arte e la natura si mescolavano alla perfezione, fatto che sarebbe stato caratteristico delle più tarde realizzazioni palladiane. Villa dei Vescovi ricevette le lodi da parte del citato Francesco Marcolini, il quale nella lista delle dediche a Alvise Cornaro precedente il IV libro di Regole generali di architettura di Sebastiano Serlio, scriveva:

Chi vuol fare un palazzo da principe pur fuor della terra vadi a Luvignano dove conteplarà uno albergo degno d' esser habitato da un pontefice o da uno imperatore nonché da ogn' altro prelato o signore ${ }^{33}$.

Bisogna in questo contesto ricordare le parole del precedentemente citato Łukasz Górnicki che descrive così il luogo dove avvenivano gli incontri degli umanisti polacchi:

Tuż u Krakowa jest rzeczka Prądnik, nad którą Samuel Maciejowski, Krakowski Biskup i Kanclerz Koronny, włoskim kształtem dom piękny zmurować kazał33.

Teniamo ancora una volta a sottolineare che il modello di questi possedimenti era, tra gli altri, la villa di Luvigliano che i polacchi avevano avuto modo di ammirare, perlomeno durante le visite alle residenza estiva di Petrarca a Arquà.

Durante i numerosi lavori di costruzione Cornaro, che nonostante il suo grande interesse verso l'ambito dell'architettura, rimaneva comunque un dilettante, aveva di fianco a sé un amico nella persona di Giovanni

33 F. Marcolini, Introduzione a: Regole generali di architettura, di Sabastiano [!] Serlio Bolognese, sopra le cinque maniere de gli edifici: cioè, thoscano, dorico, ionico, corinthio, e composito, con gli essempi dellantiquita, che per la maggior parte concordano con la dottrina di Vitruvio, con nove additioni et castigationi dal medesimo auttore in questa terza edittione fatte, come nella seguente carta è notato, Venetia 1544, vol. 4, p.1.

34 Ł. Górnicki, Dworzanin polski..., cit., pp. 62-63, trad. it: «Qui alle porte di Cracovia cè il fiumiciattolo Prądnik, nei pressi del quale Samuel Macejowski, vescovo di Cracovia e Cancelliere della Corona, fece edificare una bella casa in stile italiano». 
Maria Falconetto ${ }^{35}$, autore della loggia e dell'odeon, che ebbe modo di costruire nella residenza del mecenate a Padova. Grazie a lui il palazzo di Luvigliano acquistò bellezza e armonia. Non possiamo qui dimenticare l'informazione, secondo noi di una certa rilevanza, che egli fu anche autore degli affreschi che decorano la Sala dello Zodiaco a Palazzo d'Arco a Mantova ${ }^{36}$. Sulle quattro pareti di una delle più grandi stanze sono rimasti i segni dello zodiaco con in primo piano dei lavori che si riferiscono ciascuno ai singoli mesi. Le rappresentazioni sono completate da un dipinto sullo sfondo che si collega al mito oppure alla storia, estrapolato dalla letteratura classica ${ }^{37}$. Rammentiamo tale lavoro in quanto legato a Zwierciadło di Mikołaj Rej. A quanto sembra l’opinione diffusa riguardo alle dette similitudini delle opere di Rej con Zodiacus vitae di Palingenius ${ }^{38}$ va completata con le notizie sul lavoro della famiglia Cor-

35 Descrivendo la sua vita, Giorgio Vasari (Le vite dei più eccellenti pittori, scultori e architetti, p. 220) in primo piano mette il contatto tra Falconetto con Pietro Bembo e dopo con Alvise Cornaro: «Ma finalmente, rassettate le cose, se n'andò a Padoa, dove fu prima conosciuto e poi molto favorito da monsignor reverendissimo Bembo, che poco appresso lo fece conoscere al magnifico Messer Luigi Cornaro, gentiluomo viniziano d'alto spirito e d'animo veramente regio, come ne dimostrano tante sue onoratissime imprese».

36 Lattribuzione degli affreschi a Giovanni Falconetto la dobbiamo a Giovanni Fiocco; vedi G. Fiocco, Le architetture di Giovan Maria Falconetto, «Dedalo», 11 (1931), pp. 1203-1241; G. De Tervarent, Les fresques zodiacales du palais d'Arco à Mantoue, «Académie Royale de Belgique. Bullettin e la Classe des Beaux 'Arts», 45 (1963), pp. 244-265; G. Schweikhart, Un artista veronese di fronte all'antico. Gli affreschi zodiacali del Falconetto a Mantova, in: Roma e l'antico nell'arte e nella cultura del Cinquecento, a cura di M. Fagiolo, Roma 1985, pp. 461-488; L. Venier, Falconetto: astrologia e cultura antiquaria, in: Piranesi e la cultura antiquaria gli antecedenti e il contesto atti del convegno, 14-17 novembre 1979, Roma 1983, pp. 111-131; L. Capodieci, C. Ilari, I segreti del tempo. Prime considerazioni sullo Zodiaco di Palazzo d'Arco, «Storia dell'Arte», 87 (1996), pp. 141-167.

37 L' opera di Falconetto non era la prima in questo genere. Basta citare gli affreschi di Palazzo Schifanoia a Ferrara (1469), nelle parti ispirate alla scoperta del poema di Marcus Manilius (Marcus Manilius; I sec. D.C..) Astronomica, sugli affreschi di Pinturicchio realizzati su richiesta del papa Alessandro VI Borgia in Vaticano, oppure delle opere che si trovano nella Sala dei Pontefici, o infine sul ciclo della Sala Farnesina (oggi Galatea) nel palazzo Agostina Chigi (1466-1520).

38 Zodiacus vitae, pulcherrimum opus atque utilissimum Marcelli Palingenii Stellati [Pietro Angelo Manzoli; ca. 1500-1543], poetae ad illustrissimum Ferrariae ducem, Herculem secundum, foeliciter incipit, Venezia [1531?]. 
naro. Ancor di più per il fatto risaputo che suo genero, Bartolomeo Ridolfi, marito dell'ultima delle sei figlie, aveva lavorato in Polonia. Giorgio Vasari ritiene che il committente di questi lavori fu Spytek Jordan:

\begin{abstract}
Afferma il Palladio architetto rarissimo non conoscere persona né di più bella invenzione, né che meglio sappia ornare con bellissimi partorienti di stucco le stanze di quello che fa questo Bartolomeo Ridolfi il quale fu, non sono molti anni passati, da Spitech Giordan, grandissimo signore in Pollonia appresso al re, condotto con onorati stipendii al detto re di Pollonia, dove ha fatto e fa molte opere di stucco, ritratti grandi, medaglie e molti disegni di palazzi et altre fabriche, con l'aiuto d'un suo figliuolo che non è punto inferiore al padre ${ }^{39}$.
\end{abstract}

In verità Karol Estreicher sospetta che Vasari confuse Ridolfi con Bartolomeo Berrecci ${ }^{40}$, ma non bisogna completamente rifiutare la testimonianza delle Vite. Ricordiamo che Spytek da Zakliczyn (Wawrzyniec Jordan, 1518-1568) che gestiva numerose istituzioni in Polonia ai tempi di Sigismondo Augusto, era un famoso amante dell'arte. Nonostante i numerosi contatti con credenti di altre religioni, egli rimase cattolico per tutta la vita. Nelle sue residenze di Myślenice e Mogilany, secondo il modello italiano, si attorniò di persone d'arte e cultura. Probabilmente proprio a Mogilany vennero concepiti Zwierciadto (primavera del 1567) e anche il III libro di Żywot człowieka poczciwego di Mikołaj Rej (1505-1569), ritenuto il padre della letteratura polacca. Non è comunque escluso che l'ispirazione di Rej potrebbe essere stata l'arredamento, purtroppo non conservatosi, della residenza di campagna oppure i disegni che Ridolfi avrebbe portato con sé in Polonia. I possedimenti di Spytek sono una delle più importanti testimonianze dell'influenza che la cultura delle residenze di campagna ha avuto sulla scrittura. Purtroppo del palazzo-parco di Mogilany, realizzato negli anni 1550-1560 non è rimasta alcuna traccia ${ }^{41}$.

39 G. Vasari, Vite de' più eccellenti pittori, scultori e architetti, t. 9, Venezia 1828, parte 1, pp. 226-227.

40 Vedi G. Vasari, Żywoty najsławniejszych malarzy, rzeźbiarzy i architektów, trad. K. Estreicher, t. 5, p. 222, e t. 1, Warszawa 1985, p. XXXVIII.

41 Kochanowski era l’autore dell'aneddoto su Spytek. Vedi:. Apoftegmata: «Tytuł wielki, dochód mały»: «Spytek Jordan, kasztelan krakowski, mówiąc w radzie o do- 
Il legame tra le ville di campagna con la letteratura realizzata sotto il loro tetto è un fatto conosciuto. Guarino da Verona (Guarini) fu uno dei primi umanisti che ha richiamato dall'antichità l'idea della villeggiatura già nell'anno 1410. Nelle sue lettere scritte a Valpolicella, esalta la villa come "teatro di un refrigerio dell'anima e del corpo", dove l'aria fresca, le bellissime vedute e la fertilità della terra influiscono positivamente sul proprio benessere $^{42}$. Vale la pena inoltre ricordare che nella scuola degli umanisti veronesi si studiavano le opere di Virgilio, qui era stato scoperto Teocrito, mentre Tito Vespasiano Strozzi e Matteo Maria Boiardo iniziarono a scrivere le prime ecloghe ${ }^{43}$. In questo contesto è importante sottolineare che negli studi più recenti differenti riferimenti all'idea dell'idillio agreste e alla residenza di campagna parte della cultura del territorio veneziano si riscontrano in Jan Kochanowski, il più importante poeta del rinascimento polacco $^{44}$. Vale rammentare un testo di Kochanowski che non riguarda direttamente la tematica delle residenze extraurbane. Esso fa parte delle prime opere del poeta pubblicate dopo il suo ritorno definitivo in Polonia, verso la fine del 1558 oppure all'inizio del 1559. L' opera Satyr albo Dziki

ległościach, które wysokie urzędy za sobą niosą, też to powiedział: „Co sobie Spytek nagotuje, to mu pan krakowski zje”», trad. it.: «Spytek Jordan, castellano di Cracovia, parlando al consiglio delle patologie procurate da alti incarichi, disse così: "Quello che Spytek si prepara da mangiare, quello gli mangia il signor di Cracovia"». Su Spytek Jordan Piotr Myszkowski lanciò una nota nel suo diario, scrivendo la data del 12 marzo 1568: «Spytek Jordan de Zakliczyn, castellanus cracoviensis, moritur, vir acri ingenio et rerum peritia clarus»; vedi Dziennik biskupa Piotra Myszkowskiego 1555-1568, a cura di Ł. Kurdybacha, «Kwartalnik Historyczny», 47(1933), 3, p. 467.

42 Vedi U. Pfisterer, Donatello und die Entdeckung der Stile 1430-1445, München 2002, p. 175.

43 Vedi W.L. Grant, Neo-Latin Literature and the Pastoral, Chapel Hill 1965, pp. 118-119; L. Walther, Die Borsias des Tito Strozzi, München 1977, p. 28; A. Tissoni Benvenuti, La restauration humaniste de l'eclogue: l'école guarinienne a Ferrare, in: Le genre pastoral en Europe du XV $V^{e}$ au XVII siècle. Actes du Colloque International tenu à Saint-Etienne du 28 septembre au 1er octobre 1978, Saint Étienne 1980, pp. 25-33.

44 Dedico a questa tematica molto spazio nei capitoli: «”Pavan, an?”, czyli o jednym $\mathrm{z}$ aspektów życia teatralnego» [“„Pavan, an?”, ovvero su uno degli aspetti della vita teatrale»]; «Selvadego, czyli Dziki Mąż na renesansowym dworze oraz Santa villeggiatura, czyli kultura renesansowego ogrodu w Czarnolesie» [«Selvadego ovvero l'uomo selvaggio pressol a corte reale, oppure la Santa villeggiatura, ovvero la cultura del giardino rinascimentale di Czarnolas»], vedi M. Lenart, Patavium, Pava, Padwa..., cit. 
Mąż, pubblicata per la prima volta probabilmente nel 1563, era da molto tempo uno dei molti quesiti a cui avevano cercato di trovare risposta gli studiosi della produzione letteraria di Jan Kochanowski da Czarnolas. Una questione ancora non risolta era la fonte dell'idea di rendere il satiro come un maestro di moralità, così come la conclusione definitiva se si trattasse di un testo solo da leggere, oppure, come si sospettava, anche destinato alla presentazione in una qualche forma teatrale ${ }^{45}$. Gli studi sulla storia del teatro veneziano, nei cui avvenimenti si evidenzia la produzione letteraria di Angelo Beolco (conosciuto anche come Ruzante) introducevano la possibilità di intraprendere nuove strade nella ricerca di risposte sulle questioni finora irrisolte. Soprattutto si rende più comprensibile il riferimento al Satiro o alluomo selvaggio, la cui presenza sulla scena del teatro veneziano (anche di strada) non era nulla di eccezionale. Durante il rinascimento la figura dell'uomo selvaggio, arrivata direttamente dalla mitologia medievale e dalle leggende delle comunità che abitavano nelle Alpi non è stata rigettata, bensì si è evoluta, mescolandosi sempre più al mondo classico, tanto amato dagli umanisti. Proprio in questa unione si decise il suo destino sulla scena, sul quale un forte ruolo ebbero i cambiamenti della società che coinvolsero il territorio della Serenissima. L' arrivo nelle città di una massa di contadini, caratteristica della prima metà del XVI secolo, fu uno degli elementi che assicurò lunga vita ai personaggi della letteratura classica che vivevano ai confini del bosco, così come alla tradizione popolare. Il carattere di massa del fenomeno si legava all'improvviso bisogno di scaricare le tensioni attraverso un processo di "civilizzazione" di chi arrivava sulla strada della ricerca della chiave per una comprensione reciproca. Gli abitanti degli spazi mitici, che vivevano a stretto contatto con la natura, si prestavano perfettamente come interpreti di entrambe le realtà che rimanevano tra in loro in contrasto. La disunicità di questi personaggi e la loro "mescolanza" sono una sorte di riflesso del mondo nel quale essi apparvero. Così come all'ambiente dell'erudito si prestava meglio il satiro, così il popolo era legato ai racconti degli uomini selvaggi. Iniziano a scambiarsi i ruoli, mentre lo spazio del dialogo diventa una rappresentazione teatrale messa in scena anche in strada, e nelle sempre maggiori scene provvisorie. Oltre allo scontro della mitologia classica con il medioevo in questo periodo ci incontriamo con l' esempio della comparsa di santi eremiti in pose e figure non diverse dagli uomini selvaggi.

45 Ivi, p. 66. 
Come leggiamo nel titolo, il protagonista creato da Kochanowski possiede una doppia personalità: quella del satiro e quella dell'uomo selvaggio. Cacciato dalla foresta dai boscaioli, mostra, nel suo lungo discorso, in quali punti sono criticati problemi di base del funzionamento del paese dei satiri, mentre il marito selvaggio critica per esempio il funzionamento del parlamento, della magistratura e dell'istruzione. Espone anche un' opinione negativa sulla situazione politica e si lamenta riguardo alla decadenza della tradzione cavalleresca. Nella sua orazione non mancano riferimenti alla questione delle abitudini ma anche dell' etica, mostrando che la morale degli abitanti e le buone abitudini sono il fondamento dell'esistenza del paese.

Da molto tempo si sospettava che la citata pubblicazione potesse essere la trasposizione di una vera rappresentazione realizzata davanti al re. Queste supposizioni hanno trovato conferma soltanto nella cultura del teatro del rinascimento, in particolare nelle opere di Angelo Beolco. Ci si riferisce qui soprattutto a due testi di questo autore che si presentava in scena e scriveva con lo pseudonimo di Ruzante: Prima orazione (1521) e Seconda orazione (1528). Di una certa rilevanza è che esse vennero recitate nella loggia di Barco a Altivole, a cui vale la pena dedicare alcune osservazioni. Barco fa parte delle più antiche residenze extraurbane, create come luogo di caccia e di ricerca dei piaceri che offre il periodo estivo; ciò avvenne grazie agli sforzi fatti in tale direzione da Caterina Cornaro, regina di Cipro che abitava nel vicino castello di Asolo, non consono alle sue esigenze. Il termine barco (oppure barcho) dalla fine del Trecento significava un terreno recintato, composto da campi e bosco collegato a un giardino; tale terreno doveva assolvere al compito di riserva di caccia ${ }^{46}$. E possibile che l'idea di Pietro Bembo al quale si attribuisce il termine Barco utilizzato per la residenza di Caterina (con la quale era unito da un vincolo di parentela), venne preso dalla residenza estiva della famiglia Montefeltro, oggi non più esistente. In ogni caso Bembo viene anche ricordato per la contorta prova di unione di tale termine con il corrispondente greco della parola paradiso ${ }^{47}$. L' equivalente latino doveva essere, secondo il dotto letterato, un leporarium (latino lepus - 'lepre'). Alla prima realizzazione appartenevano i giardini, oggi non

46 Vedi L. Dami, Il giardino italiano, Roma 1924, p. 41.

47 Vedi L. Piovesan, Il barco nelle testimonianze dei biografi asolani di Caterina Cornaro, in: Il barco di Altivole contributi per la conoscenza, a cura di T. Marson, L. Piovesan, Treviso 2000, pp. 25-26. 
più esistenti, nei quali Pietro Bembo ambientò l'azione del suo Asolani (vi descrive Barco come un giardino circondato da mura e diviso in quattro parti da alcune pergole). I tre dialoghi sull'amore pubblicati da Aldo Manuzio a Venezia nell'anno $1505^{48}$ (in tre tomi successivi) mostravano prima l'amante infelice che nega l'amore, poi l'amante felice che polemizza con il predecessore, e infine l'ultimo dei disputanti che smonta le tesi espresse in precedenza ed espone la teoria dell'amore platonico come contemplazione della bellezza ideale nelle cose terrestri. Tutta l'opera viene chiusa dal racconto di un vecchio eremita il quale sviluppa davanti al lettore la dottrina dell'amore ispirata all'ascetismo cristiano che porta definitivamente alla totale mancanza di interesse verso la bellezza terrestre mentre il desiderio diventa unicamente quello del bello divino.

Inoltre la lingua - Bembo utilizza la lingua del Boccaccio, quindi il fiorentino letterario ${ }^{49}$ - così come anche lo strato ideologico dellopera, vengono in seguito messe a confronto nel teatro di Ruzante, il quale tocca le storie d'amore tra i contadini (piene di desiderio originale e di piaceri puramente fisici) con il reale mondo della popolazione che abita nelle vicinanze di Padova. È proprio in questo teatro che gli attori deridono l'ideale di amore platonico e la lingua dei dotti letterati. Ciò è stato osservato da Tadeusz Ulewicz dopo la lettura degli Asolani scrivendo di «zaskakująco tu zbieżnych z Satyrem roztrząsaniach socjologiczno-kulturalnych» ${ }^{50}$, in realtà non si riferisce al testo di Bembo, ma alle due Orazioni, che vennero presentate a Barco, e anche i testi che seguirono sulla scia del loro enorme successo. Vennero attribuiti alla tradizione gogliardico-maccheronica, in accordo alla quale si effettuavano dei discorsi su modello delle declamazioni recitate durante le cerimonie ufficiali che avevano luogo in città oppure all'università $^{51}$. Il fatto che all'orazione a Barco recitò un uomo acculturato

48 P. Bembo, Gli Asolani di messer..., Venetia 1505. La seconda edizione, con tanti rifacimenti, fu pubblicata a Venezia: De gli Asolani di M..., ne quali si ragiona d'amore primo-terzo libro, Vinegia 1530.

49 Un’ampia bibliografia sul tema Asolani la propone Storia letteraria d'Italia. Nuova edizione, a cura di A. Balduino, 1: Il Cinquecento. La dinamica del rinnovamento (1494-1533), a cura di G. Da Pozzo, vol. 1, Milano 2007, pp. 439-440, 483.

50 T. Ulewicz, O „Satyrze” Jana Kochanowskiego oraz historycznoliterackich kłopotach z bohaterem tytułowym, in: Literatura, komparatystyka, folklor. Księga poświęcona Julianowi Krzyżanowskiemu, Warszawa 1968, p. 124.

51 G. Padoan, La commedia rinascimentale veneta (1433-1565), Vicenza 1982, p. 77. 
(impersonando il contadino Beolco) davanti a unaltra persona anch' essa acculturata è già di per sé comico. Questa forma ironica nascondeva in realtà un tema serio: il valore del mondo strettamente legato alla natura, mondo rappresentato dal contadino padovano, un omaccione gioviale, ma contrario al roesso mondo, ovvero al degradato mondo della città che si allontana sempre più dalla natura ${ }^{52}$. Il successo de La prima orazione influenzò la preparazione della seconda orazione, che Ruzzante presentò alcuni anni più tardi alla presenza di Francesco, fratello di Marco Cornaro (morto nel 1524). Le circostanze erano simili. Il 7 giugno del 1528 Francesco Cornaro ricevette la nomina cardinalizia. Il Cardinale, poco tempo dopo la fine della cerimonia solenne, si presenta a Barco, vicino a Asolo, e Ruzante durante il banchetto recita, naturalmente modificando nel suo stile il dialetto padovano, La seconda Orazione. Dal tono della Seconda Orazione, si può intuire che con il nuovo cardinale non cề un'intesa come con il fratello defunto con il quale scherzava in tutta libertà ${ }^{53}$. Anche la situazione nella campagna padovana era molto cambiata, in quanto tale ambiente in quel momento soffriva una pesante fame, per questo i contadini non impersonificavano più, come nella precedente orazione, il desiderio animalesco di riempirsi lo stomaco. Il contadino smette di essere l'esempio di cui si serve Ruzante nelle polemiche letterarie e culturali. Del resto l'autore riceve la simpatia della popolazione proveniente dalle campagne che a lungo avrebbe ricordarto che nei suoi testi il loro mondo non si riduce unicamente a uno sfondo folcloristico.

Mettendo a confronto i discorsi di Ruzante con Il Satiro o l'uomo selvaggio di Kochanowski, possiamo immediatamente notare che ciò che unisce le orazioni improvvisate si trova nella presentazione, da parte di una persona importante e acculturata, di seri problemi nell'utilizzo del travestimento. Partecipando alla rappresentazione siamo confusi, ma rapidamente arriviamo alla conclusione che tutti prendiamo parte a qualche mascherata di corte. Non senza motivo, in un dato momento, il Satiro di Kochanowski si rivolge agli astanti dicendo

52 G. Padoan, Momenti del rinascimento veneto, Padova 1978, p. 106 («Medioevo e Umanesimo», 31).

53 Vedi G. Padoan, Introduzione, in: Angelo Beolco il Ruzante, Dialoghi. La seconda Oratione. I Prologhi alla Moschetta, testo critico, tradotto ed annotato, a cura di G. Padoan, Padova 1981, p. 2 («Medioevo e Umanesimo», 43). 
Gdy z was maszkarę zdejmą, a ludzie doznają, Że Polacy przodków swych barzo zostawają ${ }^{54}$.

Il fatto che il satiro di Kochanowski fosse rappresentato alla corte reale e non in una residenza extraurbana, cambia solamente gli spazi del funzionamento dei fenomeni che dipendono dalla cultura della corte rinascimentale, per la quale la ricerca di un equilibrio tra ciò che è vero e ciò che è falso, riguarda anche la riflessione sulle differenze tra il mondo della natura e il mondo interno organizzato secondo le regole ritenute troppo razionali. Del resto tra i testi del poeta polacco si trovano anche delle piccole opere come: Dryas Zamchana, conosciuta nelle versioni latine e polacche, e Pan Zamchanus, conosciuto nella sua versione latina ${ }^{55}$. Kochanowski ha realizzato Dryas per l'arrivo alla residenza extraurbana che serviva come luogo di caccia dal nome Zamch, proprietà del re Stefan Batory. Questa visita fu la conseguenza di un invito a caccia, inviato da Jan Zamojski al monarca. Ricordiamo che il proprietario dei beni e della residenza apparteneva ai più influenti "Padewczycy" e il suo busto e il suo stemma decorano ancora oggi l'ingresso e uno dei muri dell'Aula Magna di palazzo del Bo, la più antica sede dell'Università di Padova. All'inizio di maggio, fatto confermato dalle note del poeta rivolte al signore, gli ospiti, con a capo il re, ascoltarono due panegirici poetici, sebbene non sappiamo se lo stesso poeta fosse presente. Non lascia dubbi il fatto che lomaggio reso da Driada e il signore aveva la forma di una rappresentazione teatrale, fatto già intuito in precedenza, sulla base dei particolari scenici presenti nelle didascalie ${ }^{56}$ e le nostre osservazioni riguardanti il Satiro non fanno altro che confermarlo.

54 Trad. it: «Se vi tolgono la maschera la gente riconsce che i polacchi i loro antenati li abbandonano».

55 Condivido l'opinione dell'editore di Dryas, che Kochanowski pirma preparò la versione latina, e soltanto in un secondo tempo quella polacca. Vedi A. Gorzkowski, Wstęp a: Jan Kochanowski, Dryas Zamchana Polonicae et Latine. Pan Zamchanus Latine et Polonice, ed. e introduzione a cura di A. Gorzkowski, Pan Zamchanus, trad. di E. Buszewicz, Kraków 2002, pp. 8-9; Idem, Bene atque ornate. Twórczość łacińska Jana Kochanowskiego w świetle lektury retorycznej, Kraków 2004, pp. 203-213.

56 Vedi B. Baranowska, Przekład czy parafraza? „Dryas Zamchana” i „Driada Zamechska” Jana Kochanowskiego, «Meander», 55 (2000), 4, p. 368; A. Krzewińska, Sielanka staropolska: jej początki, tradycje i główne kierunki rozwoju, Warszawa 1979, pp. 99-92. 
I testi di Kochanowski succitati vennero scritti quando il poeta era legato alla corte reale. Nell'anno 1574 egli decise di ritornare presso i suoi possedimenti in campagna. Qui bisogna sottolineare un altro importante elemento, che mette in evidenza il metodo della rappresentazione dei problemi che ci interessano. In Polonia la cultura delle ville extraurbane non si potè sviluppare in una scala paragonabile alla Repubblica di Venezia in virtù delle differenze geografiche e di comunicazione. La nobiltà polacca era conosciuta per il suo legame verso le proprietà che di solito erano molto lontane dai centri urbani e poco collegate tra loro. Nelle condizioni della realtà polacca le enormi distanze erano da percorrere soprattutto su strada e non con le barche come nel caso delle Repubblica di Venezia, che nella Pianura padana disponeva di uno sviluppato sistema di vie acquatiche, permettendo un comodo trasferimento da Venezia e anche dalle città che facevano parte del suo territorio, verso i possedimenti terrieri dei quali il centro era costituito dalle ville.

Il poeta nella sua decisione di allontanarsi dalla vita della città e da quella della corte, fatto che si legava al ritorno in pianta stabile al patrimonio cittadino e alla sua amministrazione, esponeva probabilmente in modo radicale la comprensione per la cultura con la quale si imbattè in Italia, soprattutto nel territorio della Serenissima. In altre parole, su questa decisione, legata a una matura riflessione sul tema del proprio destino, un'influenza decisiva avrebbero potuto averla anche i principi di vita e del pensiero intellettuale di eminenti rappresentanti della cultura rinascimentale, osservate con cura durante i soggiorni padovani e apprezzati in seguito, un determinato periodo della propria vita ${ }^{57}$. In queste circostanze Czarnolas si presenta nel suo completo significato di possedimento extraurbano, imitando in un certo qual modo le esemplari residenze di Alvise Cornaro, Pietro Bembo oppure i fratelli Barbaro, e il suo proprietario si presenta simile a loro, in quanto raffinato amministratore. La differenza consisteva solamente nel fatto che le ville italiane erano un posto di fuga temporaneo, mentre i beni di Kochanowski erano diventati lo spazio in cui lambiente famigliare e stabile venne riempito con la costante presenza

57 In altro modo la pensa J. Sokolski, Sub tegmine tiliae. Arkadyjskie otia Jana Kochanowskiego, in: Staropolskie Arkadie, a cura di J. Dąbkowska-Kujko, J. Krauze -Karpińska, Warszawa 2010, pp. 23-24 («Studia Staropolskie. Series Nova», t. XXIX $(\mathrm{LXXXV)})$. Vale la pena sottolineare l' esatezza della tesi, profondamente analizzata nell'articolo che «l' obiettivo a cui Kochanowski aspirava era la vita contemplativa e il legame con otium honestum» (ivi, p. 28). 
dell'umanista polacco che si era formato principalmente in Italia. L' allora santa masserizia (da massaro - signore dai possedimenti di campagna), come la si era destinata a Czarnolas, descritta da Leon Battista Alberti nel 1441, nell'opera Della famiglia, con il tempo chiamata santa agricoltura ${ }^{58}$, contiene la parola santa, il che sottolinea l'allora significato di occuparsi della tenuta agricola. In questa scelta di vita sono fortemente presenti elementi morali e culturali e il suo significato sul territorio polacco non si esaurisce con le ricerche riguardanti la tradizione della proprietà terriera polacca. Tale decisione non dipendeva soltanto dallimitazione di altri popoli, in particolare dei modelli italiani. La comprensione di un reciproco mescolamento di elementi stranieri e locali permette di apprendere la realizzazione della corte di Czarnolas, che insieme altri luoghi dello stesso tipo abitati da umanisti come Spytek Jordan, oppure il vescovo Piotr Myszkowski, stimolava l'immaginazione e influenzava sensibilmente lo sviluppo e l'affermarsi di una concezione coerente del sarmatismo.

Czarny las, allora selva oscura, costituisce un importante elemento culturale dello sfondo reale con il quale si imbattè Kochanowski nel rinascimento italiano. Osserviamo come molte attenzioni siano state dedicate all'interpretazione del bosco, di cui si parla nei primi versi della Divina commedia di Dante; nellenciclopedia dedicata a questopera ${ }^{59}$, selva oscura è quindi una delle idee di base la cui corretta interpretazione permette di cogliere il senso dei molti luoghi del poema visionario del maestro italiano. Come osservano gli interpreti della Divina Commedia, il bosco è un luogo in cui il protagonista si perde durante un viaggio terrestre quando si trova in un momento importante della sua vita, ovvero ha 35 anni ed entra in una tappa successiva (Convivio, IV XXIII 6 i 13). Questo periodo è cronologicamente convergente con fatti storici, esattamente con l'anno 1300 e gli scontri durante Calendimaggio a Firenze, quando la popolazione della città si stava preparando ai balli e alle feste legate con la celebrazione della primavera. Quell' episodio politico, legato alle celebrazioni di tale festa, viene considerato l'inizio della lotta per il potere tra le frazioni dei bianchi e i dei neri Guelfi, che portò alla divisione degli abitanti della città in due fazioni nemiche. Proprio nel 1300

58 G. Benzoni, Letteratura di agronomia e di economia della villa: dalla „santa masserizia” alla „santa agricoltura”, in: La letteratura di villa e di villeggiatura. Atti del Convegno di Parma, 29 settembre - 1 ottobre 2003, Roma 2004, pp. 241-256.

59 M. Sabbatini, E. Ragni, «Selva», in: Enciclopedia Dantesca, 5, Roma 1976, pp. 137-142. 
Dante aveva compiuto 35 anni e dovette andare in esilio, fatto questo che diede inizio a un altro periodo della sua vita.

Forse per Kochanowski la corrispondenza del nome Czarnolas ['Bosconero, n.d.t.] con il topos riscontrabile nella cultura italiana, attivo non solo in un contesto dantesco (selva oscura) aveva un qualche legame; non ci permettiamo di confermarlo con certezza. Richiamamo alla mente dei futuri commentatori della vita del poeta polacco questo quesito e questo legame che si riferisce alla vita di Dante, in quanto, come giustamente osserva Janusz Pelc:

Tymczasem zaś - i jestem o tym głęboko przekonany - jest to sprawa nie tylko bardzo ważna, lecz ponadto, co jest rzeczą najbardziej tu istotną, pozwalająca nam wniknąć w tajniki kształtowania się języka poetyckiego, jakim posługiwał się Jan Kochanowski, jaki w polszczyźnie, dla dalszego rozwoju polszczyzny, tworzył. Czy Jan Kochanowski żył więc i pisał w Czarnolesie czy w Czarnym Lesie? A pytanie to powinno brzmieć - moim zdaniem - w sposób właściwszy: co Jan Kochanowski pisał w Czarnolesie, co zaś tworzył w Czarnym Lesie? ${ }^{60}$

Possiamo completare l'approfondita conoscenza dellesperto di Kochanowski con una successiva domanda ancora più specifica: se gli amici del poeta, in particolare quelli della cancelleria reale, nel formulare "w Czarnym Lesie"61 vi leggevano solamente il nome dei beni, dove si era trasferito Kochanowski, oppure si tratta di una velata allusione e un programma artistico? Il nome si può analizzare non solo dal punto di vista geografico e linguistico, ma anche da quello simbolico e letterario come scelta di un luogo specifico nel quale testo e natura creano insieme un racconto sulla vita, l'amore e la perfezione interpretata nei differenti

60 J. Pelc, $W$ „Czarnolesie” i w „Czarnym Lesie”, in: Jan Kochanowski. Interprea tacje, a cura di Jan Błoński, Kraków 1989, p. 215, trad. it.: «In questo momento sono profondamente convinto che sia una faccenda non solo molto importante, ma fattore ben più prezioso, che ci permette di approfondire i segreti della costruzione della lingua polacca, che usava Jan Kochanowski, quale ha creato all'interno della lingua polacca per un ulteriore sviluppo della stessa. Kochanowski visse e scrisse a "Bosconero" o nel "Bosco Nero"? La domanda dovrebbe suonare, secondo me, in maniera corretta: che cosa Jan Kochanowski scrisse a "Bosconero", e che cosa invece creò nel "Bosco nero"?».

61 «Nel bosco nero» (n.d.t.). 
gradi di significato; simbolicamente, come fuga dai contrasti, dagli intrighi, dagli esili che permette una realizzazione personale.

La villa è l'intermediario tra il caos (che dilaga oltre la selva oscura) e lordine riconquistato nuovamente. Questo luogo prescelto, sistematizzato e differenziato dagli altri, nel caso di Kochanowski avviene anche con il nome dato al luogo, Czarny Las (il bosco nero). Non ci si può quindi dimenticare ancora di un piccolo particolare, che per la cultura delle ville rinscimentali risulta assai importante, appunto quello dei loro nomi. Essi non venivano scelti a caso, ciò viene confermato da Barco a Altivole. Petrarca chiamò Linterno la villa nelle vicinanze di Certosa di Garignano, in ricordo di Linternum in Campania, luogo di soggiorno degli ultimi anni di vita di Scipione l'Africano il Vecchio ${ }^{62}$. Una raccolta specifica di nomi legati al bosco si trova in Italia, proprio nel territorio di Padova e dintorni. Già nel medioevo si annoveravano: la Selva di S. Maria, La Selva di Porpora, Villa del Bosco, Selvazan, Bosco di Ruban, Bosco di Carpaneda, Boschigiano, Cà del Bosco, Legnaro, Frassene, Carpane, Saliceto ${ }^{63}$.

Riassumendo dobbiamo sottolineare ancora una volta che la forma dello spazio della villa extrurbana, soprattutto nella sua fase iniziale, dove realizzò un'idea vicina agli umanisti del rinascimento, è strettamente legata con le opere letterarie. La famosa opera Hypnerotomachia Poliphili del Dominicano Francesco Colonna, pubblicata in un' edizione curata nei minimi dettagli presso la stamperia di Aldo Manuzio il vecchio (Venezia 1499), capolavoro dell'arte editoriale ${ }^{64}$, si presenta in questo caso come un' opera modello, ma non unica. Si evince soprattutto dal fatto che la realtà dello spazio del possedimento terriero si prestava più facilmente a modifiche rispetto a quella della città, chiusa all'interno delle mura, con le tracce delle piazze e delle vie. Nei tempi in cui il mondo cittadino e quello della campagna si compenetrano a vicenda

62 Vedi A. Bellani, Del vero sito della villa del Petrarca presso Milano, «Rivista Europea. Gornale di Scienza Morali, Letteratura ed Arti» (1845), fasc. di novembre-dicembre, II semestre, p. 712.

63 F.S. Dondi dall'Orologio, Dissertazioni sopra l'istoria ecclesiastica di Padova... Dissertazione prima, Padova 1802, p. 90.

64 Por. M. Lenart, W kreggu symboliki humanistycznej. Szkic o poszukiwaniu znaczeń i odkrywaniu sensu na granicy snu, in: Humanitas. Projekty antropologii humanistycznej, I: Paradygmaty - tradycje - profile historyczne, a cura di A. Nowicka-Jeżowa, Warszawa 2010, pp. 205-249 («Humanizm. Syntezy», 1). 
nel senso della lingua e della cultura (caratteristica della Serenissima nella prima metà del XVI secolo), entrambi gli ambienti vengono trattati come un simbolico sfondo. Si osserva negli Asolani di Pietro Bembo e nell'opera Cortigiano (1525) di Pietro Aretino ${ }^{65}$. Gli autori e le loro opere rappresentano la cultura della corte anche se le azioni si svolgono in campagna, oppure se obbligano ai protagonisti di parlare in volgare. Nel caso delle opere di Kochanowski si tratta di mostrare la residenza di campagna come il posto che costituisce l'ispirazione all'opera, restando allo stesso tempo una naturale scena teatrale, che unisce questo spazio con la raffinata cultura della corte rinascimentale.

A questo punto arriviamo alla conclusione che senza una comprensione dell'idea delle residenze rinascimentali extraurbane sulla base di significati risultanti dall'interpretazione dei fenomeni teatrali e letterari a esse legati, risulta difficile prevedere la misura dei reciproci paragoni tra l'Italia e la Polonia. Sembra che le personalità famose dell'umanesimo polacco che erano accomunate dalla comune esperienza padovano-veneziana abbiano elaborato, in modo abile e originale, la cultura delle ville venete facendo proprio più il carattere comunicativo rispetto a quello formale-artistico. Come creazione dipendente dal mondo delle idee durò così a lungo, come solo possibile era la reciproca comprensione tra persone che vi avevano trovato valori e riferimenti comuni. Del resto lo stesso riguarda l'ambiente degli umanisti italiani che hanno dato un tono alle concezioni e ai modelli legati con la formazione dello spazio extraurbano, dove la villa costituiva il punto di riferimento nei differenti livelli di significato.

La durata del modello artistico a cui si attingeva permetteva una lunga vita alla villa veneta nella consapevolezza culturale delle persone abituate alla sua presenza. Sembra comunque che il livello di questo fenomeno, spesso abbassato, richieda una riflessione più profonda il cui allettante obiettivo non è solo l'avvicinamento di due culture differenti, ma soprattutto le scoperta delle fonti comuni e, a volte, di aspirazioni diverse.

65 Ulteriori informazioni a riguardo in: F. Sberlati, Villania e cortesia. L’opposizione tra città e campagna dal Medioevo al Rinascimento, in: La letteratura di villa e di villeggiatura. Atti del Convegno di Parma, 29 settembre-1 ottobre 2003, Roma 2004, pp. 110-113. 


\section{Bibliografia}

Andrea Palladio e la villa veneta da Petrarca a Carlo Scarpa, Vicenza, Museo Palladio in palazzo Barbaran da Porto, 5 marzo-3 luglio 2005, a cura di G. Beltramini, H. Burns, fotografie delle ville di P. Guidolotti, Venezia 2005.

Andreoni A., Benedetto Varchi all'Accademia degli Infiammati. Frammenti inediti e appunti sui manoscritti, «Studi Rinascimentali», 3 (2005), pp. 29-44.

Baranowska B., Przekład czy parafraza? „Dryas Zamchana” $i$ „Driada Zamechska” Jana Kochanowskiego, «Meander», 55 (2000), 4, pp. 367-381.

Barycz H., Kulturalna działalność Piotra Kmity, «Rocznik Towarzystwa Przyjaciół Nauk w Przemyślu», t. 6, 1925, pp. 3-72.

Bellani A., Del vero sito della villa del Petrarca presso Milano, «Rivista Europea. Gornale di Scienza Morali, Letteratura ed Arti» (1845), fasc. di novembre-dicembre, II semestre, pp. 707-718.

Bembo P., De gli Asolani di M. ..., ne quali si ragiona d'amore primo-terzo libro, Vinegia 1530.

Bembo P., Gli Asolani di messer..., Venetia 1505.

Benzoni G., Letteratura di agronomia e di economia della villa: dalla „santa masserizia” alla „santa agricoltura”, in: La letteratura di villa e di villeggiatura. Atti del Convegno di Parma, 29 settembre-1 ottobre 2003, Roma 2004, pp. 241-256 .

Capodieci L., Ilari C., I segreti del tempo. Prime considerazioni sullo Zodiaco di Palazzo d'Arco, «Storia dell'Arte», 87 (1996), pp. 141-167.

Ćwikliński L., Klemens Janicki poeta uwieńczony (1516-1543), Kraków 1893.

Ćwikliński L., O wawrzynie doktorskim i poetyckim Klemensa Janickiego, Kraków 1919.

Da Mosto A., L' Archivio di Stato di Venezia. Indice generale storico, descrittivo e analitico, Roma 1937.

Dami L., Il giardino italiano, Milano 1924.

De Tervarent G., Les fresques zodiacales du palais d'Arco à Mantoue, «Académie Royale de Belgique. Bullettin e la Classe des Beaux 'Arts», 45 (1963), pp. 244-265 .

Dondi dall'Orologio F.S., Dissertazioni sopra l'istoria ecclesiastica di Padova... Dissertazione prima, Padova 1802-1817.

Dziennik biskupa Piotra Myszkowskiego 1555-1568, a cura di Ł. Kurdybacha, «Kwartalnik Historyczny», 47 (1933), nr 1, pp. 447-468.

Falconi G., La nuova, voga, et dilettevole villa, Milano 1559.

Fiocco G., Le architetture di Giovan Maria Falconetto, «Dedalo», 11 (1931), pp. 1203-1241.

Gallo A., Le dieci giornate della vera agricoltura di, Venezia 1566.

Gorzkowski A., Bene atque ornate. Twórczość łacińska Jana Kochanowskiego w świetle lektury retorycznej, Kraków, „Księgarnia Akademicka” 2004. 
Górnicki Ł., Dworzanin polski, a cura di R. Pollak, Wrocław 1954.

Grant W.L., Neo-Latin Literature and the Pastoral, Chapel Hill, University of North Carolina Press 1965.

I Prologhi alla Moschetta, testo critico, tradotto ed annotato, a cura di G. Padoan, Padova 1981.

Ianicius C., poetae laureati, Carmina, edidit, prefatione instruxit, annotationibus illustravit L. Ćwikliński, Cracoviae 1930 («Corpus Antiquissimorum Poetarum Poloniae Latinorum usque ad Ioannem Cochanovium», 6).

Kochanowski J., Dryas Zamchana Polonicae et Latine. Pan Zamchanus Latine et Polonice, ed. e introduzione a cura di A. Gorzkowski, trad. di E. Buszewicz, Kraków 2002.

Kotarski E., Klemens Janicki, in: Pisarze staropolscy. Sylwetki, a cura di S. Grzeszczuk, Warszawa 1991, pp. 377-406.

Krzewińska A., Sielanka staropolska: jej początki, tradycje i główne kierunki rozwoju, Warszawa 1979, pp. 99-92.

Krzywy R., Podróże do nowej Arkadii. Wizja Włoch w elegiach Klemensa Janicjusza, «Terminus», XIII (2011), fasc. 24, pp. 87-100.

L'Università di Padova. Otto secoli di storia, a cura di P. Del Negro, Padova 2002.

Lenart M., Epitaphium Cretcovii świadectwem kontaktów padewskich Jana Kochanowskiego z otoczeniem Alvisa Cornara?, in: Twórczość Jana Kochanowskiego w kontekście nowołacińskiej literatury europejskiej i polskiej, a cura di G. Urban-Godziek, Kraków 2010, pp. 64-75.

Lenart M., Lazzaro Bonamico - „buon'amico con tutti” - i jego relacje z Polakami, in: Przyjaźń w kulturze staropolskiej, a cura di A. Czechowicz, M. Trębska, Lublin 2013, pp. 117-126 («Studia i Materiały do Dziejów Literatury Wczesnonowożytnej», 2).

Lenart M., Patavium, Pava, Padwa. Tło kulturowe pobytu Jana Kochanowskiego na terytorium Republiki Weneckiej, Warszaw 2013 («Studia Staropolskie. Series Nova», tom XXXIII (LXXXIX)).

Lenart M., Studenti polacchi a Padova e loro mecenatismo in patria, in: Mecenati, artisti e pubblico nel Rinascimento. Atti del XXI Convegno Internazionale Chianciano Terme-Pienza, a cura di L. Secchi Tarugi, Firenze 2011, pp. 501-511 (Quaderni della Rassegna, 68).

Lenart M., W kręgu symboliki humanistycznej. Szkic o poszukiwaniu znaczeń i odkrywaniu sensu na granicy snu, in: Humanitas. Projekty antropologii humanistycznej, I: Paradygmaty - tradycje - profile historyczne, a cura di A. NowickaJeżowa, Warszawa 2010, pp. 205-249 («Humanizm. Syntezy», 1).

Lollio A., Lettera in laude della villa, Ferrara 1540 (Venetia 1544).

Manzoli P.A., Zodiacus vitae, pulcherrimum opus atque utilissimum Marcelli Palingenii Stellati, poetae ad illustrissimum Ferrariae ducem, Herculem secundum, foeliciter incipit, Venezia [1531?].

Marcolini F., Introduzione a: Regole generali di architettura, di Sabastiano [!] Serlio Bolognese, sopra le cinque maniere de gli edifici: cioè, thoscano, dorico, 
ionico, corinthio, e composito, con gli essempi dellantiquita, che per la maggior parte concordano con la dottrina di Vitruvio, con nove additioni et castigationi dal medesimo auttore in questa terza edittione fatte, come nella seguente carta è notato, Venetia 1544.

Maylender M., Storia delle accademie d'Italia, III, Bologna 1929.

Muraro M., Civiltà delle ville Venete. Conferenza tenuta il 23.10.1964 alla Hertziana di Roma, Venezia 1964.

Padoan G., Momenti del rinascimento veneto, Padova 1978 («Medioevo e Umanesimo», 31).

Padoan G., La commedia rinascimentale veneta (1433-1565), Vicenza 1982.

Pelc J., W „Czarnolesie” i w „Czarnym Lesie”, in: Jan Kochanowski. Interpretacje, a cura di J. Błoński, Kraków 1989, pp. 211-223.

Pfisterer U., Donatello und die Entdeckung der Stile 1430-1445, München 2002.

Piovan F., Per la biografia di Lazzaro Bonamico ricerche sul periodo dell'insegnamento padovano (1530-1552), Trieste 1988.

Piovesan L., Il barco nelle testimonianze dei biografi asolani di Caterina Cornaro, in: Il barco di Altivole contributi per la conoscenza, a cura di T. Marson, L. Piovesan, Treviso 2000, pp. 24-33.

Rime di m. Pietro Bembo, Milano 1808.

Sabbatini M., Ragni E., «Selva», in: Enciclopedia Dantesca, 5, Roma 1976, pp. 137-142 .

Sberlati F., Villania e cortesia. L’opposizione tra città e campagna dal Medioevo al Rinascimento, in: La letteratura di villa e di villeggiatura. Atti del Convegno di Parma, 29 settembre-1 ottobre 2003, Roma 2004, pp. 65-114.

Schweikhart G., Un artista veronese di fronte allantico. Gli affreschi zodiacali del Falconetto a Mantova, in: Roma e l'antico nell'arte e nella cultura del Cinquecento, a cura di M. Fagiolo, Roma 1985, pp. 461-488.

Sokolski J., Sub tegmine tiliae. Arkadyjskie otia Jana Kochanowskiego, in: Staropolskie Arkadie, a cura di J. Dąbkowska-Kujko, J. Krauze-Karpińska, Warszawa 2010, pp. 20-35 («Studia Staropolskie. Series Nova», t. XXIX (LXXXV)).

Storia letteraria d'Italia. Nuova edizione, a cura di A. Balduino, 1: Il Cinquecento. La dinamica del rinnovamento (1494-1533), a cura di G. Da Pozzo, vol. 1, Milano 2007.

Ślaski J., Wokół literatury włoskiej, węgierskiej i polskiej w epoce renesansu. Szkice komparatystyczne, Warszawa 1991.

Taegio B, La villa dialogo... all'inuittissimo, \& gloriosissimo imperatore Ferdinando Primo, Milano 1599.

Tissoni Benvenuti A., La restauration humaniste de l'eclogue: lécole guarinienne a Ferrare, in: Le genre pastoral en Europe $d u X V^{e}$ au XVII siècle. Actes du Colloque International tenu à Saint-Etienne du 28 septembre au 1er octobre 1978, Saint Étienne 1980, pp. 25-33. 
Ulewicz T., Lenigmatica Accademia degli studenti polacchi a Padova (negli anni 1547-1549 ca.), «Atti e Memorie dell'Accademia Patavina di Scienze, Lettere ed Arti», 100 (1987-1988), pp. 87-93.

Ulewicz T., O „Satyrze” Jana Kochanowskiego oraz historycznoliterackich kłopotach $z$ bohaterem tytułowym, in: Literatura, komparatystyka, folklor. Księga poświęcona Julianowi Krzyżanowskiemu, Warszawa 1968, pp. 113-132 .

Vasari G., Vite de' più eccellenti pittori, scultori e architetti, Venezia 1828$-1830$.

Vasari G., Żywoty najsławniejszych malarzy, rzeźbiarzy i architektów, a cura di K. Estreicher, Warszawa 1989.

Vedova G., Biografia degli scrittori padovani, Padova 1832, I, pp. 491-492.

Venier L., Falconetto: astrologia e cultura antiquaria, in: Piranesi e la cultura antiquaria gli antecedenti e il contesto atti del convegno, 14-17 novembre 1979, Roma 1983, pp. 111-131.

Vianello V., Il letterato, l'accademia, il libro contributi sulla cultura veneta del Cinquecento, Padova 1988.

Walther L., Die Borsias des Tito Strozzi. Ein Lateinisches Epos der Renaissance, München 1977.

Windakiewicz S., I polacchi a Padova, in: Omaggio dell'Accademia Polacca di Scienze e Lettere all'Università di Padova nel settimo centenario della sua fondazione, Cracovia 1922, pp. 1-34.

Windakiewicz S., Padwa. Studyum z dziejów cywilizacyi polskiej, Kraków 1891. 